\title{
Flavor violating $Z^{\prime}$ from $S O(10)$ SUSY GUT in High-Scale SUSY
}

\author{
Yuji Omura* \\ Kobayashi-Maskawa Institute for the Origin of Particles and the Universe, \\ Nagoya University, Nagoya 464-8602, Japan \\ E-mail: vujiomurdeken.phys.nagoya-u.ac.jp
}

\begin{abstract}
We propose an $S O(10)$ supersymmetric grand unified theory (SUSY GUT), where the $S O(10)$ gauge symmetry breaks down to $S U(3)_{c} \times S U(2)_{L} \times U(1)_{Y} \times U(1)_{X}$ at the GUT scale and $U(1)_{X}$ is radiatively broken at the SUSY-braking scale. In order to achieve the observed Higgs mass around $126 \mathrm{GeV}$ and also to satisfy constraints on flavor- and/or CP-violating processes, we assume that the SUSY-breaking scale is $O(100) \mathrm{TeV}$, so that the $U(1)_{X}$ breaking scale is also $O(100) \mathrm{TeV}$. One big issue in the SO(10) GUTs is how to realize realistic Yukawa couplings. In our model, not only 16-dimensional but also 10-dimensional matter fields are introduced to predict the observed fermion masses and mixings. The Standard-Model quarks and leptons are linear combinations of the 16- and 10-dimensional fields so that the $U(1)_{X}$ gauge interaction may be flavor-violating. We investigate the current constraints on the flavor-violating $Z^{\prime}$ interaction from the flavor physics and discuss prospects for future experiments.
\end{abstract}

Flavor Physics \& CP Violation 2015,

May 25-29, 2015

Nagoya, Japan

\footnotetext{
* Speaker.
} 
In this letter, we propose an $S O(10)$ supersymmetric Grand Unifield theory (SUSY GUT), where the realistic fermion masses and mixings are achieved by introducing extra 10-dimensional matter fields, based on Ref. [四]. In the minimal $S O(10)$ GUT, the SM quarks and leptons are embedded into three 16-dimensional fields []. In this case, the mass spectrum of the SM fermions are inconsistent with the experimental result, because of the matter unification. In our model, the SM quarks and leptons come from 10- and 16-dimensional fields, and especially, the righthanded down-type quarks and left-handed leptons in the SM are given by the linear combinations of 10- and 16-dimensional fields. We assume that $S O(10)$ gauge symmetry breaks down to $S U(3)_{c} \times S U(2)_{L} \times U(1)_{Y} \times U(1)_{X}\left(\equiv G_{S M} \times U(1)_{X}\right)$ around $10^{16} \mathrm{GeV}$ according to the nonzero vacuum expectation values (VEVs) of $S O(10)$ adjoint fields. Thus, the low-energy effective theory is an $U(1)_{X}$ extension of the SUSY SM with extra matters. The additional gauge symmetry will survive up to the SUSY scale, but we could expect that it is radiatively broken, as the electroweak (EW) symmetry breaking in the minimal supersymmetry Standard Model (MSSM). We assume that SUSY particles in the SUSY SM, except for gauginos, reside around $100 \mathrm{TeV}$, in order to realize the observed $126 \mathrm{GeV}$ Higgs mass and also to satisfy constraints on flavor- and/or CP-violating processes. The gauge coupling unification is rather improved when only the gaugino masses are around $1 \mathrm{TeV}$ []], and the dangerous dimension-five proton decay is suppressed [田]. On the other hand, since $\tan \beta$ (the ratio of the VEVs of the two Higgs doublets in the SUSY SM) is close to one, it is difficult to explain the large hierarchy between top and bottom quarks when all the matter fields are embedded into only $\mathbf{1 6}$ representational representations. In our model, the introduction of 10-representational matter fields makes it possible to explain the large hierarchy.

The mass of the $Z^{\prime}$ boson associated with the gauged $U(1)_{X}$ may be $O(100) \mathrm{TeV}$ corresponding to the SUSY scale, so that it may be viable in the searches for flavor violations. The right-handed down-type quarks and left-handed leptons in the SM are given by linear combinations of the parts of 10- and 16-dimensional fields. Thus, that generically leads to flavor-violating $Z^{\prime}$ interaction and crucial promises against flavor experiments. We will see that tree-level Flavor Changing Neutral Currents (FCNC) induced by the $Z^{\prime}$ boson are generated and they largely contribute to the flavor violation processes: for instance, $\mu \rightarrow 3 e, \mu$-e conversion in nuclei, and $K^{0}-\bar{K}^{0}$ and $B_{d / s}^{0}-\bar{B}_{d / s}^{0}$ mixings. Especially, we find that the $\mu-e$ conversion and the $\mathrm{CP}$-violation in $K$ system provide the strongest constraints with our model.

\section{Setup of $S O(10)$ SUSY GUT}

We introduce a 10-dimensional matter field in the each generation in addition to 16-dimensional matter fields. Three $S O(10)$-singlet matter fields $S_{i}$ are also introduced to achieve the realistic masses of neutrinos [四]. The matter fields $\mathbf{1 0}_{i}$ and $\mathbf{1 6}_{i}$ are decomposed as the ones in Table $\mathbb{W}$. For convenience, the assignment of $S U(5) \times U(1)_{X}$ is also shown in Table $\mathbb{W}$. The superpotential is written down according to the $S O(10)$ gauge symmetry introducing $\mathbf{1 0}_{H}, \mathbf{1 6}_{H}$ and $\overline{\mathbf{1 6}}_{H}$ Higgs fields to break the EW and $U(1)_{X}$ gauge symmetries. In addition, two $S O(10)$ adjoint Higgs fields, $\mathbf{4 5}_{H}$ and $\mathbf{4 5}_{H}^{\prime}$, are introduced in our model. They develop nonzero VEVs, and then the $S O(10)$ gauge symmetry breaks down to $G_{S M} \times U(1)_{X}$ at the GUT scale. The low-energy effective theory is the $U(1)_{X}$ extension of the SUSY SM with 10- and 16-dimensional matter fields. 


\begin{tabular}{|c|c|c|c|c|c|c|}
\hline & $Q_{L}$ & $U_{R}^{c}$ & $E_{R}^{c}$ & $\hat{L}_{L}$ & $\hat{D}_{R}^{c}$ & $N_{R}^{c}$ \\
\hline$S O(10)$ & \multicolumn{6}{|c|}{16} \\
\hline$S U(5) \times U(1)_{X}$ & \multicolumn{3}{|c|}{$(10,-1)$} & \multicolumn{2}{|c|}{$(\overline{\mathbf{5}}, 3)$} & $(\mathbf{1},-5)$ \\
\hline \multirow[t]{2}{*}{$G_{\mathrm{SM}}$} & $\left(\mathbf{3}, \mathbf{2}, \frac{1}{6}\right)$ & $\left(\overline{\mathbf{3}}, \mathbf{1},-\frac{2}{3}\right)$ & $(\mathbf{1}, \mathbf{1}, 1)$ & $\left(\mathbf{1}, \mathbf{2},-\frac{1}{2}\right)$ & $\left(\overline{\mathbf{3}}, \mathbf{1}, \frac{1}{3}\right)$ & $(\mathbf{1}, \mathbf{1}, 0)$ \\
\hline & $L_{L}^{\prime}$ & $D_{R}^{\prime c}$ & $\overline{L_{L}^{\prime}}$ & $\overline{D_{R}^{\prime c}}$ & & \\
\hline$S O(10)$ & \multicolumn{4}{|c|}{10} & & \\
\hline$S U(5) \times U(1)_{X}$ & \multicolumn{2}{|c|}{$(\overline{\mathbf{5}},-2)$} & \multicolumn{2}{|c|}{$(5,2)$} & & \\
\hline$G_{\mathrm{SM}}$ & $\left(\mathbf{1}, \mathbf{2},-\frac{1}{2}\right)$ & $\left(\overline{\mathbf{3}}, \mathbf{1}, \frac{1}{3}\right)$ & $\left(\mathbf{1}, \mathbf{2}, \frac{1}{2}\right)$ & $\left(\mathbf{3}, \mathbf{1},-\frac{1}{3}\right)$ & & \\
\hline
\end{tabular}

Table 1: Charge assignment for matter fields. Charge assignment for $G_{\mathrm{SM}}$ is denoted as $\left(S U(3)_{c}, S U(2)_{L}\right.$, $\left.U(1)_{Y}\right) . U(1)_{X}$ gauge coupling constant is normalized as $g_{X}=g / \sqrt{40}$ at GUT scale, where $g$ is $S O(10)$ gauge coupling constant.

After the $U(1)_{X}$ symmetry breaking, the chiral superfields $\hat{D}_{R i}^{c}$ and $D_{R i}^{\prime c}\left(\hat{L}_{L i}\right.$ and $\left.L_{L i}^{\prime}\right)$ mix each other, and we find the massless modes which correspond to the SM right-handed down-type quarks and left-handed leptons. Eventually, the relevant Yukawa couplings for quarks and charged leptons are described as

$$
W_{Y}^{S S M}=h_{u i j} Q_{L i} U_{R j}^{c} H_{u}+Y_{d i j} Q_{L i} D_{R j}^{c} H_{d}+Y_{e i j} L_{L i} E_{R j}^{c} H_{d}+\widetilde{\mu}_{i j}\left(\overline{D_{R h i}^{c}} D_{R h j}^{c}+\overline{L_{L h i}} L_{L h j}\right) .
$$

$D_{R i}^{c}$ and $L_{L i}\left(D_{R h i}^{c}\right.$ and $\left.L_{L h i}\right)$ are the (heavy) chiral superfields of right-handed down-type quarks and left-handed leptons in the mass bases. The Yukawa coupling constants for the SM down-type quarks and charged leptons are described as

$$
\left(Y_{d}\right)_{i j}=\left(h_{u i k}+\varepsilon_{d i k}\right)\left(\hat{U}_{D_{R}^{c}}\right)_{k j},\left(Y_{e}\right)_{i j}=\left(\hat{U}_{L_{L}}^{T}\right)_{i k}\left(h_{u k j}+\varepsilon_{e k j}\right),
$$

using the mixing parameters, $\left(\hat{U}_{D_{R}^{c}}\right)_{k j}$ and $\left(\hat{U}_{L_{L}}\right)_{i k}$, and the additional parameters, $\varepsilon_{d, e k j}$, originated from the higher dimensional parameters. Then, the realistic Yukawa couplings are realized by the mixing between $\mathbf{1 0}$ - and $\mathbf{1 6}$-dimensional matter fields.

Since the fields in $\mathbf{1 0}$ and $\mathbf{1 6}$ representations carry different $U(1)_{X}$ charges, the SM fields have flavor-dependent $U(1)_{X}$ interaction. The $U(1)_{X}$ gauge interactions of right-handed downtype quarks and left-handed leptons are described in the interaction basis as $\mathscr{L}_{g}=-i g_{X}\left(3 \overline{\hat{\varphi}}_{i} \not^{\prime} \hat{\varphi}_{i}-\right.$ $\left.2 \overline{\varphi^{\prime}}{ }_{i} Z^{\prime} \varphi^{\prime}{ }_{i}\right)$, where the factors 3 and -2 are $U(1)_{X}$ charges for the fermionic components $\hat{\varphi}_{i}$ and $\varphi_{i}^{\prime}$ of the chiral superfields $\hat{\psi}_{i}$ and $\psi_{i}^{\prime} . Z^{\prime}$ is the $U(1)_{X}$ gauge boson and $g_{X}$ is defined as $g_{X}=g / \sqrt{40}$ at GUT scale, where $g$ is the $S O(10)$ gauge coupling constant. Using the unitary matrix $U_{\psi}$, we define the flavor-violating couplings $A_{i j}^{\varphi}$ for the SM fermions as $\mathscr{L}_{g}=-i g_{X} \bar{\varphi}_{i}\left(5\left(\hat{U}_{\psi}^{\dagger} \hat{U}_{\psi}\right)_{i j}-2 \delta_{i j}\right) \not \not^{\prime} \varphi_{j} \equiv$ $-i g_{X} A_{i j}^{\varphi} \bar{\varphi}_{i} \not^{\prime} \varphi_{j}$, where $\varphi$ is the fermion component of the chiral superfield $\psi$ in the mass base and denotes right-handed down-type quark $\left(d_{R}^{c}\right)$ and left-handed lepton $\left(l_{L}\right)$. As discussed in Ref. [四], we find that all elements of the flavor violating couplings are $O(1)$, so that we need careful analyses of their contributions to flavor physics, even if the $Z^{\prime}$ boson is quite heavy.

\section{Flavor physics}

The large tree-level FCNCs involving the $Z^{\prime}$ boson are promised in our model. Here, we 
sketch the relevant constraints on the flavor-violating $Z^{\prime}$ interactions and give prospects for future experiments.

In our model, the SUSY SM Higgs doublets are charged under $U(1)_{X}$, so that their nonzero VEVs contribute to the $Z^{\prime}$ mass $\left(m_{Z^{\prime}}\right)$ as well as the SM gauge bosons. The mass mixing between $Z$ and $Z^{\prime}$ is generated by the VEVs as well. Then the $Z^{\prime}$ interaction is described by the mixing and the tree-level FCNCs. The flavor-violating couplings significantly contribute to $B_{d / s}^{0}-\bar{B}_{d / s}^{0}$ and $K^{0}-\bar{K}^{0}$ mixings, flavor-violating decays, and $\mu$-e conversion in nuclei. Below, we summarize the constraints relevant to the $Z^{\prime}$ interaction, and discuss the predictions in flavor physics.

The most relevant processes would be the $\mu \rightarrow 3 e$ process and the $\mu$-e conversion process in the lepton flavor violation. The current upper bound on the branching ratio of $\mu \rightarrow 3 e$ is $1.0 \times$ $10^{-12}$ [[]]. We find that our model is safe for the current experimental bound as long as $m_{Z^{\prime}}$ is $O(100) \mathrm{TeV}[\mathrm{W}]$. We also evaluate the branching ratios of other lepton flavor violating decays, and we find that they are also much below the current experimental upper bounds. The flavor violating coupling $A_{12}^{l_{L}}$ also gives rise to the $\mu$-e conversion process. In our model, the branching ratio for the Au target is close to the current upper bound at the SINDRUM-II [ [6]. The branching ratios for the other materials are estimated as $O\left(10^{-13}\right)$ as well, so that we expect that our model could be proved in the future experiments.

The $Z^{\prime}$ FCNCs contribute to the mass splitting and $\mathrm{CP}$ violation in neutral meson systems. The measurement of $K^{0}-\bar{K}^{0}$ oscillation is a strong probe on both real and imaginary part of $\left(A_{12}^{d_{R}^{c}}\right)^{2}$. Especially, the $\mathrm{CP}$ violation gives a sever constraint on the FCNC, so that the $Z^{\prime}$ mass has to be heavier than a few $\mathrm{PeV}$, if $A_{12}^{d_{R}^{c}}$ possesses $O(1) \mathrm{CP}$ phase.

The author would like to thank J. Hisano, Y. Muramatsu, and M. Yamanaka for fruitful collaboration. This work is supported by Grant-in-Aid for Scientific research from the Ministry of Education, Science, Sports, and Culture (MEXT), Japan, No. 23104011.

\section{References}

[1] J. Hisano, Y. Muramatsu, Y. Omura and M. Yamanaka, Phys. Lett. B 744, 395 (2015) [arXiv:1503.06156 [hep-ph]].

[2] H. Georgi, AIP Conf. Proc. 23, 575 (1975); H. Fritzsch and P. Minkowski, Annals Phys. 93, 193 (1975).

[3] J. Hisano, T. Kuwahara and N. Nagata, Phys. Lett. B 723, 324 (2013) [arXiv:1304.0343 [hep-ph]].

[4] J. Hisano, D. Kobayashi, T. Kuwahara and N. Nagata, JHEP 1307 (2013) 038 [arXiv:1304.3651 [hep-ph]].

[5] U. Bellgardt et al. [SINDRUM Collaboration], Nucl. Phys. B 299 (1988) 1.

[6] W. H. Bertl et al. [SINDRUM II Collaboration], Eur. Phys. J. C 47, 337 (2006). 